\title{
Barriers and Facilitators to Expanding Roles of Medical Assistants in Patient-Centered Medical Homes (PCMHs)
}

\author{
Jeanne M. Ferrante, MD, MPH, Eric K. Shaw, PhD, Jennifer E. Bayly, BS, \\ Jenna Howard, PhD, M. Nell Quest, PhD, Elizabeth C. Clark, MD, MPH, and \\ Connie Pascal, MS
}

Background: Many primary care practices participating in patient-centered medical home (PCMH) transformation initiatives are expanding the work roles of their medical assistants (MAs). Little is known about attitudes of MAs or barriers and facilitators to these role changes.

Methods: Secondary data analysis of qualitative cross-case comparison study of 15 New Jersey primary care practices participating in a PCMH project during 2012 to 2013. Observation field notes and in-depth and key informant interviews (with physicians, office managers, staff and care coordinators) were iteratively analyzed using grounded theory.

Results: MA roles and responsibilities changed from a mostly reactive role, completing tasks dependent on physician orders during the patient visit and facilitating patient flow through the office, to a more proactive one, conducting previsit planning, engaging in the overall care for patients, and assisting with population management. MAs differed in their attitudes about increased responsibilities, with some welcoming the opportunity to take on expanded roles, others resenting their increased responsibilities, and some expressing insufficient understanding regarding why new tasks and procedures were being implemented. Major barriers to MA role shifts included 1) insufficient understanding of the PCMH concept, 2) lack of time for added responsibilities, 3) additional workload without additional compensation, 4) disparate levels of medical knowledge and training, 5) reluctance of clinicians to delegate tasks, 6) uncertainty in making new workflow changes routine, 7) staff turnover, and 8) change fatigue. MAs were more positive about their role shifts when they 1) understood how their responsibilities fit within broader PCMH practice transformation goals; 2) received formal training in new tasks; 3) had detailed protocols and standing orders; 4) initiated role changes with small, achievable goals; 5) had open communication with clinicians and practice leaders; and 5) received additional compensation or paths to career advancement.

Conclusions: Practice leaders need to be conscious of obstacles when they increase expectations of MAs, and they must be willing to invest time and resources into developing their MA workforce. An environment that allows open dialog with MAs and rewards and compensation that recognizes their increased efforts will help make expansion of MA roles occur more smoothly and efficiently. ( $\mathrm{J}$ Am Board Fam Med 2018;31:226-235.)

Keywords: Grounded Theory, New Jersey, Patient-Centered Care, Primary Health Care

Patient-centered medical home (PCMH) transformation initiatives are widespread, and many programs are expanding the roles of medical assistants

This article was externally peer reviewed.

Submitted 15 August 2017; revised 10 November 2017; accepted 30 November 2017.

From the Department of Family Medicine and Community Health, Rutgers Robert Wood Johnson Medical School, New Brunswick, NJ (JMF, JH, ECC); Institute for
(MAs) to implement team-based care and add capacity in primary-care practices. ${ }^{1}$ For example, MAs are tasked to screen patients for risky behav-

Health, Health Care Policy and Aging Research, New Brunswick (JMF); Rutgers Cancer Institute of New Jersey, New Brunswick (JMF); Department of Community Medicine, Mercer University School of Medicine, Savannah, GA (EKS); Preliminary Medicine, Rutgers Robert Wood 
iors $^{2}$, track lab reports ${ }^{3}$, identify preventive and chronic care gaps ${ }^{3-5}$, outreach to patients for cancer screenings ${ }^{6}$, call patients after emergency department visits or hospitalizations ${ }^{3}$, reconcile medications ${ }^{7}$, scribe clinical encounters in electronic medical records ${ }^{1}$, offer HIV screenings ${ }^{8}$, and administer vaccinations per protocol. ${ }^{5}$ They also serve as health coaches to improve lifestyle behaviors9, chronic disease care processes ${ }^{3,10-18}$, and medication adherence. ${ }^{19}$ These innovative MA roles have been reported across diverse settings, including private practices ${ }^{2,3,5,20}$, community health centers $5,10,13,15,16,19$, academic primary-care practices ${ }^{8,9,12,14}$, rural practices ${ }^{17}$, and large health care systems. $^{4,5,7}$

Outcomes in studies that have focused on or included MA role expansion or innovation have varied. Some pilot programs have found improvements in cancer screenings ${ }^{4,6}$, cholesterol levels ${ }^{21}$, diabetes control ${ }^{11,21}$, medication adherence ${ }^{19}$, physical activity levels and body mass index. ${ }^{9}$ However, Ferrer and colleagues ${ }^{2}$ found that there was poor adoption of MA health coaching, and several randomized trials testing the effects of MA diabetes health coaches found no significant differences in hemoglobin A1C levels or hospitalizations between control and intervention group patients. ${ }^{10,13,15,18}$ There is currently a limited understanding as to why there was success in some studies and not others.

The majority of published research on this topic has described expansion of MA roles for specific disease-oriented programs, such as health coaching for patients with diabetes. ${ }^{10,13-16}$ To our knowledge, only 3 studies have specifically evaluated MAs' expanding roles in the broader context of primary-care transformation projects. ${ }^{3,5,20}$ Ladden et $\mathrm{al}^{5}$ described the changing roles and responsibilities of MAs in exemplar primary care practices that

Johnson Medical School, Piscataway (JEB); Rutgers Center for Law and Justice, Newark (MNQ); Rutgers School of Communication and Information, New Brunswick (CP).

Funding: This research was supported through a grant from Horizon Healthcare Services, Inc. (1469-JMF).

Conflict of interest: none declared.

Acknowledgement: Portions of this manuscript have been presented at the 42nd North American Primary Care Research Group Annual Conference, New York, NY, November 21-25, 2014.

Corresponding author: Jeanne M. Ferrante, MD, MPH, 112 Paterson Street, New Brunswick, NJ 08901 (E-mail: jeanne.ferrante@rutgers.edu). implemented workforce innovations, but they did not report any barriers to these changes. Cronholm et al. ${ }^{20}$ and Naughton et $\mathrm{al}^{3}$ described several challenges to expanding roles of MAs, such as MA resentment regarding lack of extra pay for their extra work, MAs not understanding the PCMH concept, and physicians' concerns about lack of MA training and skills. Naughton et $\mathrm{al}^{3}$ also reported reluctance of providers in delegating tasks to MAs and sustainability challenges, as well as some strategies used to overcome these barriers. ${ }^{3}$ Both the Cronholm and Naughton studies ${ }^{3,20}$ evaluated early transformation processes of a Pennsylvania multipayer Chronic Care Initiative program that started in $2008^{22}$, and they conducted interviews with clinicians, staff, and administrators 2 to 4 years after program implementation. Therefore, subjects' responses may have been affected by recall bias. Little is known about MAs' own perspectives regarding their new and changing roles in primary care. Having a clearer understanding of how MAs view and feel about new PCMH endeavors that affect their roles and relationships, can provide insights into an understudied area of PCMH transformation. The objectives of our research are to describe how the roles of MAs evolved as part of a PCMH transformation project in New Jersey, to report MAs' attitudes regarding their new responsibilities, and to identify barriers and facilitators to the MA role changes.

\section{Methods}

\section{Setting and Sample}

This observational study is a secondary analysis of data collected from a qualitative cross-case comparison of 15 community primary-care practices in the NJ Primary Care Research Network that were participating in one of the first single-payer PCMH demonstration projects in New Jersey during 2012 to $2013 .{ }^{23}$ All practices attained at least Level I PCMH recognition from the National Committee for Quality Assurance before participation. The 15 sites represented a purposive diverse sample (in size, location, and patient population) out of 31 total primary care practice sites participating in the payer's second wave of the PCMH program. All 15 practices were located in New Jersey, privately owned, and consisted of 1 to 8 physicians (average, 5). By participating in the project, practices received the following: 1) Educational tools (play- 
book of strategies, webinars, conference calls) to help them improve health quality and utilization metrics; 2) a registered nurse serving as a population care coordinator (PCC) or a subsidy to pay for a PCC; 3) quarterly reports of the practice's quality and utilization metrics data; and 4) extra revenues, including per member per month payments and end-of-year incentive bonuses for achieving metric goals. MAs were not specifically targeted by the PCMH program. The Institutional Review Board at Rutgers Robert Wood Johnson Medical School approved this study.

\section{Data Collection and Management}

The purpose of the parent study was to perform an independent qualitative evaluation of the $\mathrm{PCMH}$ demonstration project. Our goal was to provide real-time insights into the PCMH transformation process of the participating primary-care practices and to assess the usefulness of the project's intervention strategies. Our research team consisted of 2 family physician researchers (JF, EC), 2 sociologists (JH, ES), a cultural anthropologist (NQ), and a communication and information specialist (CP). None of the research team members worked at the practices. Three researchers collected data $(\mathrm{JH}$, $\mathrm{CP}, \mathrm{NQ}$ ); each assessed 4 to 6 practices. Data were collected at each practice site during the first few months of program implementation and 1 year later, and included direct observations over approximately 4 days at each practice site captured in fieldnotes; in-depth, recorded and professionally transcribed semistructured in-person interviews with each practice's office manager, medical director, and PCC; and key informant interviews with MAs, and other clinicians and office staff. The medical director for each practice signed consent for the practice to participate in the study. All semistructured interviews were voluntary, and a separate informed consent was obtained for each interview. All MAs in each practice were observed in their daily work $(\mathrm{N}=58)$, and key informant interviews were conducted with 54 MAs (ranging from 2 to 8 per site). Two practices (under same ownership) did not use any MAs. In those practices, we included the lead physician's perceptions regarding MAs in our findings. No compensation was provided to participants.

Observation templates and interview guides were developed using a review of the PCMH literature and our prior knowledge and experience with
PCMH transformation efforts, and they were modified and refined as needed after initial observations. Study researchers, who conducted key informant interviews (with MAs and others) and observed practice activities, handwrote detailed fieldnotes including some verbatim quotes while at the practice site, and they later (typically the same day) typed up the complete fieldnote in a Word document. Approximately 1000 pages of deidentified observation fieldnotes and transcriptions were imported into Atlas.ti (Atlas.ti Scientific Software Development GmbH, Berlin, Germany), a qualitative data management software program, for coding and analysis.

\section{Analysis}

For the parent study, we analyzed data iteratively using a grounded theory approach that consisted of a series of immersion/crystallization cycles. ${ }^{24}$ Initially, the research team met on a weekly basis to review the data, reading and coding jointly line by line to define preliminary codes and agree on emerging topics. This same consensus process was used to refine and finalize the prelminary codebook as new data were available. Individual team members subsequently coded data independently, with at least 2 members coding all data. Coding discrepancies were reviewed at team meetings and resolved through consensus. Data within codes were read and analyzed in a second immersion/crystallization cycle, and emerging themes and interpretations were identified. A third immersion/crystallization cycle was used to refine themes and interpretations and identify negative or disconfirming evidence for our themes. During this process, the changing roles of MAs emerged as a theme. For this article, we conducted a secondary analysis wherein we reread and analyzed all data referencing MAs. We used an immersion/crystallization cycle in which coded data segments related to MAs were reread to identify themes and patterns among the fieldnotes and interviews. At this stage, themes emerged regarding the role of MAs, the barriers encountered, and strategies used to expand their roles. As is common with qualitative analysis, we compared and contrasted each fieldnote/interview with our emergent themes. ${ }^{25}$ If data from an observation or interview did not fit into an existing theme, this prompted the development of an additional theme. As our themes continued to crystallize by finding additional evidence from participants across the practices, we 
Table 1. Changes in Medical Assistant Tasks Implemented during PCMH Demonstration Project

\begin{tabular}{|c|c|}
\hline $\begin{array}{l}\text { Before PCMH Project } \\
\text { (Facilitation of patient flow through office) }\end{array}$ & $\begin{array}{l}\text { After PCMH Project Implementation } \\
\text { (Patient care/population management) }\end{array}$ \\
\hline $\begin{array}{l}\text { Call and escort patients to examination room } \\
\text { Take and record vital signs and chief complaint }\end{array}$ & $\begin{array}{l}\text { Obtain family and social histories, reconcile medications and } \\
\text { chronic problems lists, document cancer screenings }\end{array}$ \\
\hline $\begin{array}{l}\text { Give injections, draw blood, perform EKGs or other } \\
\text { procedures per physician order during patient visit }\end{array}$ & $\begin{array}{l}\text { Pre-visit planning (chart reviews, huddles) } \\
\text { Carry out standing orders and protocols (give immunizations, } \\
\text { provide mammogram orders/lab forms, refill routine } \\
\text { medications) }\end{array}$ \\
\hline $\begin{array}{l}\text { Give out patient education handouts per physician } \\
\text { request during patient visit }\end{array}$ & $\begin{array}{l}\text { Proactively hand out patient education materials (eg, smoking } \\
\text { cessation) } \\
\text { Conduct patient education using motivational interviewing (eg, } \\
\text { weight, diabetes) } \\
\text { Organize/conduct patient awareness campaigns to address } \\
\text { preventive care, eg, through telephone calls, wearing buttons on } \\
\text { shirts, mailings }\end{array}$ \\
\hline $\begin{array}{l}\text { Call for results as per physician request during } \\
\text { patient visit }\end{array}$ & $\begin{array}{l}\text { Gather documentation of preventive services, other metrics, } \\
\text { consultations, and hospitalization reports prior to patient visit }\end{array}$ \\
\hline Give reports that come into office to physicians & $\begin{array}{l}\text { Track, manage and enter data into electronic medical record/other } \\
\text { data management tools }\end{array}$ \\
\hline File charts & Assist with risk stratification of patients \\
\hline
\end{tabular}

EKG, electrocardiography; PCMH, patient-centered medical home.

gained greater insights into thematic commonalities and variations. We also explored and report here those instances where there was little evidence of a particular theme across all practices, but were reported by different members (MAs, nurses, office managers, or clinicians) of a few practices. Doing so provides information that may be helpful for other practices and valuable for future research. The quotations included below represent and illustrate our key findings.

\section{Results}

Data from the first few months of program implementation revealed the many barriers and negative attitudes encountered when practices tried to expand roles of the MAs. By 1 year later, most practices had largely overcome these barriers by using the strategies outlined in the Recommendations section below.

\section{Changes in MA Activities}

MA roles and responsibilities changed over the course of this study in all practices, but not in the same way or extent. All MAs reported that before participating in the $\mathrm{PCMH}$ demonstration project, their main responsibilities included facilitating patient flow, taking patients from the waiting room to the examination room, and helping them exit the office. In addition, before the PCMH project, MAs mostly worked reactively, evidenced by their descriptions of tasks that were dependent on what the physician ordered during each patient visit. With participation in the PCMH demonstration project, MA roles changed in a variety of ways (see Table 1 ). Some became more proactive and engaged in the overall care for patients in the office, as well as assisting with population management of patients not scheduled for office visits. For example, after PCMH implementation, some MAs began conducting more thorough patient intakes, such as obtaining family and social histories, reconciling medications, screening for obesity or smoking status, and documenting cancer screenings and immunizations. MAs in some practices sought out and were trained in motivational interviewing to provide patient education regarding weight, exercise, smoking cessation, and low-sodium diets. Other practices used MAs to conduct outreach calls or send letters to patients not seen in the office but who were due for preventive or chronic care screening and tests.

\section{MA Attitudes Regarding their Changing Roles}

We found varying MA attitudes in the different practices regarding their expanded roles. Some welcomed the opportunity to be more engaged. As 1 MA described her role to the study researcher: 
She (MA) is now responsible for collecting more data from the patients and that makes her feel more accomplished as a MA...[however], "we (MAs) never go into rooms with the doctors...If we could work with the doctors more that would make our jobs more interesting... a more team approach would be good...maybe the doctors could use us more." She adds that not all the doctors are on the same page with what the MAs should be doing, and it would be good if they could all get on the same page because, "we'd like to do more." (fieldnote, Practice 11)

Similarly, another MA voiced her satisfaction with doing more in the office:

She (MA) likes feeling that she is making the doctors' jobs a little easier. She admitted that she felt her role as MA was somewhat underused in the past. She said she has worked here for 14 years, and she knew she was capable of doing more than they had her doing. (fieldnote, Practice 10)

Another MA commented to the researcher that she enjoyed learning more about clinical conditions:

[MA] mentions how much she likes [PCC] and how $[\mathrm{PCC}]$ is always giving them interesting little things to read- "she has cool stuff, interesting stuff to learn, like the info on body mass index and the WebMD stuff on diabetes." (fieldnote, Practice 9)

Conversely, other MAs resented the increased responsibilities and felt stressed by time constraints.

I (researcher) asked how [MA] feels about all the changes to her job lately. She lowered her voice and said, "It is just that there's so much to do in so little time. It is a lot for 1 person."; She said it is especially hard when patients are difficult. "Sometimes they get nasty when we ask them all these questions." (fieldnote, Practice 10)

MAs in some practices had limited understanding of the PCMH concept and how their tasks assisted with population care management. For example, the study researcher described:

In cases where office staff members were involved in the entering of data, they often came to understand the $[\mathrm{PCMH}]$ project as being only about data entry. One MA expressed this attitude quite succinctly when she said of the project, "It is all about documentation, not transformation." (fieldnote, Practice 5)

Some MAs expressed a cynical viewpoint of the $\mathrm{PCMH}$ as noted by a researcher:

[MA] has been with the practice for 6 years. Her understanding of the PCMH idea is that the "patients come into the doctor's office so the insurance company can save money on hospitalization. It is about the money factor - the less they go into the hospital, the reimbursement is bigger." (fieldnote, Practice 15)

Below, we describe barriers at the MA and physician/office levels that contributed to cynicism and resentment of MAs regarding their extra responsibilities, as well as facilitators of more positive attitudes in MAs.

\section{Barriers to MA Role Changes}

MA Barriers to Role Shifts

Our evidence points to several barriers specific to MAs, including insufficient understanding of the PCMH concept, concerns about lack of time for increased responsibilities, additional workload without additional pay, and lack of medical knowledge. Table 2 includes sample quotations exemplifying these barriers.

In some practices, MAs stated they had insufficient communication from practice leaders about the PCMH concept, so they tended to view all their new responsibilities as busywork, and they did not understand the philosophy of PCMH. In addition, many MAs made comments about having to multitask and felt they lacked time for all these added responsibilities. Some noted they could no longer organize their work in the patient flow structure as previously done, and more flexibility during their work day was needed. In addition, some MAs expressed they were doing a nurse's job without being paid as one. Finally, MAs had disparate levels of experience and training, with certification courses having lasted anywhere from 6 weeks to a year or more. Several MAs required extensive on-the-job training to increase clinical knowledge. In some instances, a lack of MA experience or knowledge was perceived to impact practice functions and achievement of PCMH goals. 


\begin{tabular}{ll}
\hline Barriers & Sample Quotations \\
\hline $\begin{array}{l}\text { Insufficient MA understanding } \\
\text { of the PCMH concept }\end{array}$ & $\begin{array}{l}\text { I (researcher) asked if there is ever conversation between her (MA) and the doctors about the } \\
\text { [PCMH program]. She said no...she wishes they talked about it more in this practice. She } \\
\text { feels that the MAs understand their new tasks, but she doesn't feel that they understand the } \\
\text { "PCMH or any of the pilots." Her understanding of the concept of the PCMH is "to have } \\
\text { the patient feel comfortable and confident that we will take care of all their medical needs." } \\
\text { (fieldnote, Practice 10) }\end{array}$
\end{tabular}

[The MA] knew about the metrics and was able to name BMI and smoking as examples. She said, "It's not really a change but now we do things $100 \%$ of the time"... like taking someone's BMI every time they come in which is, "kinda dumb...especially for acute visits." (fieldnote, Practice 9)

Lack of time for added responsibilities

[The MA] gets concerned sometimes when more tasks are added "because more responsibility means that it takes longer." She said that when 4 clinicians are working and there's only one MA rooming, it can get very stressful. (fieldnote, Practice 15)

Additional workload without additional pay

Lack of MA knowledge or training

[The PCC] has heard that there has been some turnover and there is good amount of frustration within the ranks of the MAs. Apparently they haven't had raises in 3 years and the demands of the job are increasing-they are citing this kind of work as an example of something that is adding to their workload. (fieldnote, Practice 9)

One thing $[\mathrm{PCC}]$ is frustrated by is that she feels for the most part, the MAs are just "memorizing tasks and not really thinking." She (PCC) has been surprised by the low level of clinical knowledge that the MAs have. For instance, she said that one MA asked her if a high PT/INR meant that it is thick or thin. [The PCC] said that when she was talking to MAs about the metrics, one of them said, "Good luck getting patients to get a colonoscopy," and someone else said (regarding colonoscopy), "What? Does [insurance company] want patients to live forever?" (fieldnote, Practice 10)

[If I could change one thing] I think that it's getting the medical assistants to think more like doctors, and to kind of function without being told, doing things without being told... we have mostly medical assistants, they don't really think like nurses. They're not that trained...nurses think differently, but they cost a lot more money.... I think the medical assistants try, but they just don't have that knowledge base enough to kind of anticipate...So that's the price we pay for hiring lower trained people. And I guess that you can train them. They're reasonably smart; they're not dumb. But, it takes a lot of work. (physician interview, Practice 9)

Reluctance to delegate tasks to MA

[A nurse practitioner commented that] the MA shouldn't be allowed to do the patient's health maintenance (ie, fill the standing orders that the health maintenance screen says the patient needs). "They just see it as a checklist and they treat it that way. It's not a checklist!" She explains that if a patient has not gotten a test or followed up on something they've been told to follow up on, then just re-ordering probably isn't going to help. She said that health maintenance "should be a conversation." (fieldnote, Practice 4)

Uncertainty on how to make workflow changes more routine

[Handing out smoking cessation handouts] was not part of the routine initially; it was new and it wasn't getting done all the time... [The health plan] was good at telling us what they wanted us to do, but not really how to do it. You know, they wanted these metrics, they wanted these reports, they wanted...but there wasn't a lot of help. We were trying to figure it out ourselves. (physician interview, Practice 1)

Staff turnover

One office manager described this as bittersweet. An MA of eight years who had trained in the practice since her internship had gradually been entrusted with increased responsibilities, developed skills, and become a practice leader. Based on the competencies the practice had nurtured in her, she was able to secure a new position at the local hospital that included higher compensation and tuition support for her nursing program. (fieldnote, Practice 6)

Change fatigue

The MAs worked so hard last summer-they busted their [butts] pulling charts. Now, that's all over, but then it was this iPad Depression screening project that had a 27 -page manual for them to read, and then [this PCMH project] came in - so it's just been a lot. (Clinical supervisor, Practice 9)

BMI, body mass index; MA, medical assistant; PCMH, patient-centered medical home; PCC, population care coordinator; PT/INR, prothrombin time/international normalized ratio.

\section{Practice Barriers to MA Role Shifts}

Our findings also pointed to barriers that were outside of how the MAs viewed their role shifts, but were related to practice leaders/clinicians or the practice as a system (see Table 2). In some practices, clinicians were reluctant to delegate tasks to the MA, for example, performing preventive counseling. While some physicians provided MAs with substantial room to follow standing orders and protocols without much oversight, other evidence suggested that some clinicians did not trust the MAs to recognize their boundaries and had concerns about 


\begin{tabular}{|c|c|}
\hline Facilitators & Sample Quotations \\
\hline $\begin{array}{l}\text { Explanation of how new MA } \\
\text { responsibilities fit within the } \\
\text { broader PCMH practice } \\
\text { transformation goals }\end{array}$ & $\begin{array}{l}\text { [The MA] reiterated that she thinks [Doctor], [Office Manager], and [Population } \\
\text { Care Coordinator] do a really good job of explaining exactly what they want } \\
\text { for the QI projects. She said the staff generally takes orders well, but that it's } \\
\text { easier when they understand and appreciate the goals of the project and get } \\
\text { good instructions...She has a pretty robust understanding of PCMH, about } \\
\text { care coordination, about the importance of patients getting the disease follow- } \\
\text { up and preventive care that they need, and about why they collect so much } \\
\text { data. (fieldnote, Practice 12) }\end{array}$ \\
\hline Extra training & $\begin{array}{l}\text { [The PCC] tells me that the MAs feel uncomfortable talking to a patient about } \\
\text { the patient's weight (or smoking) when they themselves (the MAs) are } \\
\text { overweight and/or smoke... [She] is coaching the MAs on how to approach } \\
\text { difficult topics with a patient.... [The office manager] also has mentioned this } \\
\text { coaching as one of the things she believes [PCC] excels in doing, and that she } \\
\text { has noticed a difference in how the MAs are working with patients. (fieldnote, } \\
\text { Practice 9) }\end{array}$ \\
\hline Detailed protocols and standing orders & $\begin{array}{l}\text { They have standing orders for chronic disease management as well, so, for } \\
\text { example, they have standing orders for diabetic labs. They use a screening } \\
\text { questionnaire before administering vaccines too. For diabetic retinal } \\
\text { screenings, they have a form they use to get the eye doctor to send back the } \\
\text { results. (fieldnote, Practice 13) }\end{array}$ \\
\hline Open communication & $\begin{array}{l}\text { The first way to make sure that staff buy-in happens is communication, } \\
\text { communication, communication...we allow the staff to ask questions, or to } \\
\text { question why we're saying we should do things this way, because no one truly } \\
\text { understands unless they see the big picture. So the staff doesn't feel that this is } \\
\text { what I do because I have to do it. Hopefully, they understand the process and } \\
\text { the reason why they're doing what they're doing. (Office Manager interview, } \\
\text { Practice 5) }\end{array}$ \\
\hline Initial small and achievable goals & $\begin{array}{l}\text { [The MA] said they had decided to start with the smaller goals first and after } \\
\text { that to work on the bigger goals. Medication reconciliation was a small goal, } \\
\text { whereas screenings will be a bigger goal. Another bigger goal is to have less } \\
\text { patients running to the emergency room, which she says they can work on by } \\
\text { encouraging better control and compliance. She gave the example of making } \\
\text { sure CHF patients take their water pills every day by instructing them on the } \\
\text { importance of doing this. (fieldnote, Practice 6) }\end{array}$ \\
\hline Compensation for extra efforts & $\begin{array}{l}\text { They have a new feedback system that goes along with the bonuses for doctors } \\
\text { and staff, part of which [the lead physician] bases on their engagement with } \\
\text { the PCMH and QI initiatives currently underway... Everybody accepts the } \\
\text { system and understands why they are doing the projects that they are doing, } \\
\text { and what role they each play in the overall plans. (fieldnote, Practice 12) }\end{array}$ \\
\hline
\end{tabular}

MA, medical assistant; QI, quality improvement; PCC, population care coordinator; PCMH, patient-centered medical home.

the MAs' knowledge base. There was also uncertainty expressed by some physicians in how to make the new MA tasks part of their normal routine, for example, handing out smoking-cessation materials to all smokers. In addition, many practices faced staffing challenges, which placed burdens on the other staff. On the one hand, some practices had to dismiss MAs who could not accept or handle these new changes, while high-functioning MAs left for higher-paying jobs or to pursue a formal nursing degree. Finally, many of the practices were involved in multiple pilot projects at the same time, so some MAs expressed a sense of "change fatigue."

\section{Recommendations for Expanding MA Roles}

The following recommendations highlight strategies practices used to create more positive environ- ments where MAs welcomed their new opportunities. Sample quotations regarding these facilitators are listed in Table 3.

1. Explain how new MA responsibilities fit within the broader PCMH practice transformation goals: Clarify that their roles are shifting from a patient flow structure, that is, getting this patient in front of me in and out of the practice, to assistance with population care management.

2. Provide extra training. Some practices had a nurse or nurse care coordinator who provided project specific training to MAs, for example, on how to counsel patients regarding unhealthy behaviors and how to better manage chronic conditions. Some MAs needed train- 
ing on using technology and how to document and manage data. Stories about specific patients and data showing how process changes improved patient care and outcomes were very helpful for the MAs to understand the value of achieving clinical targets.

3. Create detailed protocols and standing orders. A manual with standardized protocols helped MAs know what was expected of them.

4. Provide open communication opportunities with clinicians and practice leaders. Having regular meetings with an environment where MAs felt safe to voice their opinions and concerns made them feel that they were involved in making decisions. In addition, to improve communication between physicians and MAs, several practices used the teamlet model, whereby an MA is consistently assigned to the same physician ${ }^{12}$, as well as morning huddles to proactively prepare for the patient visit and set expectations of MAs.

5. Set achievable changes and small goals initially, for example, medication reconciliation and smoking status. This gave MAs confidence and a sense of accomplishment.

6. Provide rewards for extra efforts. Additional compensation or paths to career advancement helped MAs to welcome expanded responsibilities. For example, $1 \mathrm{MA}$ was named the "Champion of Quality Measures" and another MA worked under the PCC to train new staff, perform patient outreach, and conduct officewide previsit planning. In another practice, an MA became the data manager to ensure complete documentation and to create reports. One practice provided monetary bonuses to staff members as well as physicians.

\section{Discussion}

Because MAs are omnipresent in primary care, they are often the ones tasked with new responsibilities as part of PCMH transformation and practice improvement projects. To our knowledge, we are the first to describe mixed MA attitudes toward these changes. While some welcomed the opportunity to take on expanded roles, others did not understand the importance of these newly assigned tasks and resented the increased responsibilities, which contributed to high turnover. This is similar to a study conducted in the Veterans Administration system, which found that task delegation from primary care physicians to nurses resulted in more burnout in the nurses. ${ }^{26}$ Our study offers some insights into how to make role changes more favorably viewed by MAs.

It is important for practice leaders to be conscious of potential obstacles when increasing expectations of MAs. While we qualitatively studied a relatively small sample of 15 practices in New Jersey, we found similar barriers to MA role shifts reported by others in Pennsylvania and Ohio, including resistance to implementation due to practice culture, insufficient communication from practice leaders, lack of clinician trust in MAs' competence due to their insufficient training, lack of MA engagement with the PCMH model, and MAs' frustration with added workload without additional compensation. . $^{30,27}$

To facilitate the transitioning of MAs into these expanded roles, it is paramount to develop a teamwide shared understanding of how new MA responsibilities contribute to practice transformation and to secure buy-in from staff and all clinicians. Our findings illustrate the importance of having open communication via regular meetings and establishing an environment where the MAs feel safe to voice concerns and be involved in decision making. In addition, steps need to be taken to improve the relationship and communication between physicians and MAs. Strategies previously suggested, and also used in our practices, include using a teamlet model (of the same clinician-MA dyad each day) ${ }^{12}$, which increases physician comfort level with expanded MA roles ${ }^{1}$, and having morning huddles for collaborative previsit planning. ${ }^{28}$ Martinez et al ${ }^{29}$ described a "shareport" card that facilitates face-toface meetings between physicians and MAs, providing opportunities to collaboratively develop a list of MA tasks and explore and solve problems.

Furthermore, to sustain changes to the MA role and retain MAs in practices, investments in time and resources are needed to develop the new MA workforce. This requires additional training and supervision to ensure MA tasks are within their scope of practice. The quality of MA education and training received varies widely, and no standard educational curriculum currently exists, especially for MAs to assume these new roles. ${ }^{30}$ Practices that succeeded in this regard had a motivated registered nurse who took it on herself to provide training to the MAs. Educating MA students in this new 
PCMH model may help future MAs better integrate into team-based care while decreasing strenuous workloads for office staff. ${ }^{31}$ In addition, providing financial incentives or paths to career advancement for MAs can help to overcome reluctance on their part and increase MA retention. ${ }^{13}$ Finally, dissemination of lessons learned and strategies used are needed to enable other primary care practices to expand MA roles more smoothly and efficiently.

To see this article online, please go to: http://jabfm.org/content/ 31/2/226.full.

\section{References}

1. Bodenheimer T, Willard-Grace R, Ghorob A. Expanding the roles of medical assistants: Who does what in primary care? JAMA Intern Med 2014;174: 1025-6.

2. Ferrer RL, Mody-Bailey P, Jaén CR, Gott S, Araujo S. A medical assistant-based program to promote healthy behaviors in primary care. Ann Fam Med 2009; 7:504-12.

3. Naughton D, Adelman AM, Bricker P, Miller-Day M, Gabbay R. Envisioning new roles for medical assistants: Strategies from patient-centered medical homes. Fam Pract Manag 2013;20:7-12.

4. Kanter M, Martinez O, Lindsay G, Andrews K, Denver C. Proactive office encounter: A systematic approach to preventive and chronic care at every patient encounter. Perm J 2010;14:38-43.

5. Ladden MD, Bodenheimer T, Fishman NW, et al. The emerging primary care workforce: Preliminary observations from the primary care team: Learning from Effective Ambulatory Practices project. Acad Med 2013;88:1830-4.

6. Baker AN, Parsons M, Donnelly SM, et al. Improving colon cancer screening rates in primary care: A pilot study emphasising the role of the medical assistant. Qual Saf Health Care 2009;18:355-9.

7. Reedy AB, Yeh JY, Nowacki AS, Hickner J. Patient, physician, medical assistant, and office visit factors associated with medication list agreement. J Patient Saf 2016;12:18-24.

8. Anim M, Markert RJ, Okoye NE, Sabbagh W. HIV screening of patients presenting for routine medical care in a primary care setting. J Prim Care Community Health 2013;4:28-30.

9. Djuric Z, Segar M, Orizondo C, et al. Delivery of health coaching by medical assistants in primary care. J Am Board Fam Med 2017;30:362-70.

10. Ruggiero L, Moadsiri A, Butler P, et al. Supporting diabetes self-care in underserved populations: a randomized pilot study using medical assistant coaches. Diabetes Educ 2010;36:127-31.

11. Nelson K, Pitaro M, Tzellas A, Lum A. Practice profile. Transforming the role of medical assistants in chronic disease management. Health Aff (Millwood) 2010;29:963-5.

12. Chen EH, Thom DH, Hessler DM, et al. Using the Teamlet Model to improve chronic care in an academic primary care practice. J Gen Intern Med 2010; 25(Suppl 4):S610-S614.

13. Ivey SL, Tseng W, Kurtovich E, et al. Evaluating a culturally competent health coach intervention for Chinese American patients with diabetes. Diabetes Spectr 2012;25(2).

14. Willard-Grace R, DeVore D, Chen EH, Hessler D, Bodenheimer T, Thom DH. The effectiveness of medical assistant health coaching for low-income patients with uncontrolled diabetes, hypertension, and hyperlipidemia: Protocol for a randomized controlled trial and baseline characteristics of the study population. BMC Fam Pract 2013;14:27.

15. Ruggiero L, Riley BB, Hernandez R, et al. Medical assistant coaching to support diabetes self-care among low-income racial/ethnic minority populations: randomized controlled trial. West J Nurs Res 2014;36:1052-73.

16. Van der Wees PJ, Friedberg MW, Guzman EA, Ayanian JZ, Rodriguez HP. Comparing the implementation of team approaches for improving diabetes care in community health centers. BMC Health Serv Res 2014;14:608.

17. MacKay FD, Anderson JE, Klein MC, Berkowitz J, MacKay JT, Gailius J. The modified medical office assistant role in rural diabetes care. Can J Rural Med 2014;19:49-56.

18. Freund T, Peters-Klimm F, Boyd CM, et al. Medical assistant-based care management for high-risk patients in small primary care practices: A cluster randomized clinical trial. Ann Intern Med 2016;164: $323-30$.

19. Thom DH, Willard-Grace R, Hessler D, et al. The impact of health coaching on medication adherence in patients with poorly controlled diabetes, hypertension, and/or hyperlipidemia: A randomized controlled trial. J Am Board Fam Med $2015 ; 28: 38-45$.

20. Cronholm PF, Shea JA, Werner RM, et al. The patient centered medical home: Mental models and practice culture driving the transformation process. J Gen Intern Med 2013;28:1195-201.

21. Willard-Grace R, Chen EH, Hessler D, et al. Health coaching by medical assistants to improve control of diabetes, hypertension, and hyperlipidemia in lowincome patients: A randomized controlled trial. Ann Fam Med 2015;13:130-8.

22. Gabbay RA, Bailit MH, Mauger DT, Wagner EH, Siminerio L. Multipayer patient-centered medical home implementation guided by the chronic care model. Jt Comm J Qual Patient Saf 2011;37: $265-73$.

23. Patel UB, Rathjen C, Rubin E. Horizon's patientcentered medical home program shows practices 
need much more than payment changes to transform. Health Aff (Millwood) 2012;31:2018-27.

24. Borkan J. Immersion/crystallization. In: Crabtree BF, Miller WL, eds. Doing qualitative research. 2nd ed. Thousand Oaks, CA: Sage Publications; 1999; 179-94.

25. Strauss A, Corbin J. Basics of qualitative research: Grounded theory procedures and techniques. Newbury Park, CA: Sage; 1990.

26. Edwards S, Helfrich C, Grembowski D, et al. Task delegation and burnout trade offs among primary care providers and nurses in Veterans Affairs Patient Aligned Care Teams (VA PACT). J Am Board Fam Med 2018;31:83-93.

27. Elder NC, Jacobson CJ, Bolon SK, et al. Patterns of relating between physicians and medical assistants in small family medicine offices. Ann Fam Med 2014; 12:150-7.

28. O'Malley AS, Gourevitch R, Draper K, Bond A, Tirodkar MA. Overcoming challenges to teamwork in patient-centered medical homes: A qualitative study. J Gen Intern Med 2015;30:183-92.

29. Martinez M, Bigney J, Jernigan J. A feedback tool to improve physician-medical assistant communication. Fam Pract Manag 2014;21:5-9.

30. Chapman SA, Marks A, Dower C. Positioning medical assistants for a greater role in the era of health reform. Acad Med 2015;90:1347-52.

31. Wilson AB, Fegan F, Romence B, Uhe K, Dionne B. Precepting the medical assistant practicum: Expectations and rewards: An evaluation of preceptors' opinions. J Allied Health 2011;40:212-5. 